\title{
Microsurgical treatment of recurrent coiled aneurysms
}

\author{
Carlos A. David, M.D. \\ Lahey Clinic Medical Center, Burlington, Massachusetts
}

The number of endovascularly-treated aneurysms has steadily increased over the past two decades. A small but significant number of recurrent or partially recanalized aneurysms have been identified. If repeat endovascular therapies are not feasible due to anatomical or technical considerations, surgical clipping may be considered. The technical challenges in the microsurgical treatment include coils interfering with the clip application; the possibility of an aneurysm that is less collapsible and less easy to manipulate; and intraluminal thrombus formation requiring thrombus and coil removal. The video presents the technical steps and nuances for clipping. The technical issues for coil removal are illustrated in two cases.

The video can be found here: http://youtu.be/NvhSDVT4iuE.

KEYWORDS aneurysm; coiled aneurysm; recurrent; microsurgery; clipping; video 